DESY 83-067

Ju1y 1983

\title{
A PRECISION MEASUREMENT OF THE T' MESON MASS
}

by

D.P. Barber, D.H. Bremer, J. Kewisch, H.C. Lewin, T. Limbern, H. Mais, G. Ripken, R. Rossmanith, R. Schmidt Deutsches Elektronen-Synchrotron DESY, Homburg

and

ARGUS Collaboration

and

Crystal Ball Collaboration

ISSN $0418-9833$ 


\title{
A PRECISION MEASUREMENT OF THE T' MESON MASS
}

\author{
D.P. Barber ${ }^{*}$, D.H. Bremer, J. Kewisch, H.C. Lewin, T. Limberg, \\ H. Mais, G. Ripken, R. Rossmanith, R. Schmidt \\ DESY, Hamburs, Germany
}

and the ARGUS - Collaboration ${ }^{\text {l) }}$

and the Crystal Ball Collaboration ${ }^{2}$ )

ABSTRACT : The $e^{+} e^{-}$- storage ring DORIS II provides polarized beams with polarizations up to $80 \%$ in the energy range of the $\mathrm{T}^{\prime}$ meson. The method of resonance depolarization allows the average beam energy to be determined to a precision of $\pm 0.1 \mathrm{MeV}$. An energy scan of the hadronic cross section over the resonance range with the detectors ARGUS and Crystal Ball determines the $T^{\prime}$ mass to be $(10023.1 \pm 0.4 \pm 0.5) \mathrm{MeV}$.

*) on leave of absence from the Massachussets Institute of Technology, Cambridge, U.S.A. 
We report here the results of a mass measurement of the $T^{\prime}$ meson using the method of resonance depolarization. This method has already been used to determine the mass of the $T$ meson ${ }^{3}$ ) at Novosibirsk. The $T^{\prime}$ experiment was performed from July 20 to July 24, 1983 at the electron-positron storage ring DORIS II. The result is of obvious spectroscopic interest. In addition, it will allow a better control of the beam energy for resonance data taking in the future.

Details of DORIS II are described elsewhere ${ }^{4)}$. The main operating characteristics for this experiment were as follows : electron and positron currents between 18 and $30 \mathrm{~mA}$, peak luminosity at $1.5 \cdot 10^{31} / \mathrm{cm}^{2} / \mathrm{sec}$, beam life times of around 3.5 hours, and beam polarizations of about $80 \%$

In electron-positron storage rings, the beam particles become polarized by the Sokolov-Ternov effect ${ }^{5}$ ). The polarization of the beams can be destroyed continuously by a weak time-dependent magnetic field 6,7). The frequency of this depolarizing field gives precise information on the mean energy of the beams as will be explained in the follotsing.

The spins of the polarized particles precess around the vertical axis. The number of spin precessions per orbit revolution is $\gamma \cdot(g-2) / 2$, where $\gamma$ is the Lorentz factor and $g$ is the magnetic moment of the electron. If a particle is kicked vertically by a radial magnetic field at a certain position of the ring, its spin is tilted forward or backward. If the radial field is time-dependent and in phase with the spin precession at the fixed ring position, the spin is rotated over many revolutions from the vertical into the horizontal direction, and the polarization is destroyed. The resonance condition for the frequency $f_{D}$ of the applied depolarizing field is

$$
\left|\frac{g-2}{2} \gamma \pm n\right| \quad f_{0}=f_{D},
$$

where $\mathrm{n}$ is any integer, $1 \mathrm{l}$ in our case, and $\mathrm{f}_{\mathrm{O}}$ is the orbit revolution frequency.

The block diagram of the DORIS depolarizer is shoun in Fig. I. The sinewave generated by a highly precise frequency synthesizer is added to the input of a power amplifier which drives the vertical feedback coil in DORIS. 
The maximum field integral of the coil is of the order of 0.1 Gauss meter. The synthesizer is swept over a frequency range, and the resulting beam polarization is measured for each sweep by the DORIS polarimeter ${ }^{8)}$.

The layout of the polarimeter is shown in Fig. 2. A circularly polarized laser beam is directed head-on against the electron beam. The vertical distribution of the backscattered photons shows an asymmetry which is proportional to the electron beam polarization. This asymmetry is measured by a leadscintillator-sand:sich shotser counter with a movable slit in front.

Fig. 3 shows the result of a typical depolarization measurement. The curve gives the degree of polarization as function of the frequency. The average beam energy is then calculated from the resonance frequency by the formula given above. A frequency interval of $\pm 0.25 \mathrm{kHz}$ corresponds to $\pm 0.1 \mathrm{MeV}$ per beam, i.e. $\pm 0.22 \mathrm{MeV}$ in the CMS energy of $\mathrm{e}^{+} e^{-}$collisions. During the energy scan described belov', different energies have been determined uith different precisions.

The width of the depolarizing resonance is much smaller than the energy spread of the beam which is caused by quantum emission. The energy of each particle in the beam fluctuates around the average energy. The time of these fluctuations is small compared to the depolarization time, therefore the depolarizer averages over the energy fluctuations.

The cross section of the reaction

$$
\mathrm{e}^{+} \mathrm{e}^{-} \rightarrow \text { hadrons }
$$

is measured by the detectors ARGUS and Crystal Ball at 13 different CMS energies in the range from 9.96 to $10.04 \mathrm{GeV}$. A total integrated luminosity of about $2.0 / \mathrm{pb}$ has been collected by each of the two detectors.

ARGUS is a nev magnetic spectrometer ${ }^{l)}$ equipped with a central drift chamber, time-of-flight scintillators, shower counters and muon chambers. The trigger conditions and the selection criteria for hadronic events are described in Ref. 1. In addition to three uell reconstructed charged particles pointing to the interaction point, a minimum total energy deposit of $1.7 \mathrm{GeV}$ in the shower counters is required. This additional requirement speeds up the 
reconstruction procedure and reduces considerably the contamination from $\tau$ pairs and from two-photon interactions. For each scan point, the number of selected hadronic events is divided by the number of Bhabha events in an angular range between about 20 and 160 degrees. This ratio determines the visible cross section shown in Fig. 4. In addition to the shown vertical error bars, which are of statistical origin only, there is an overall vertical scale error of about 10\%, which has no influence on the mass determination. The ratio of subsamples of Bhabha events has been controlled to be constant within statistics for all scan points. Beam-gas or beam-wall background contributes less than $0.5 \%$ as has been checked with several separated beam runs during the scan. The horizontal error bars take into account the different precision with which the energy is determined by depolarization for each point.

The curve in Fig. 4 is the result of a four-parameter fit to the ARGUS data using the expression of Jackson and Scharre ${ }^{9)}$ for radiative corrections. The four parameters are the $T^{\prime}$ mass, the energy spread in DORIS, the continuum level and the peak cross section. The fit takes into account cross section errors as well as energy errors, since these are slightly different for each point as mentioned above. The fit results in $\mathrm{m}\left(\mathrm{T}^{\prime}\right)=(10023.43 \pm 0.45) \mathrm{MeV}$ and a RMS width of the CMS energy of $(8.2 \pm 0.5)$ MeV. The quoted errors include the energy uncertainties on each scan point.

The Crystal Ball 2) detector consists mainly of a spherical segmented shell of $\mathrm{NaI}$ ( $\mathrm{TI}$ ) shower counters which cover 93\% of $4 \pi$ solid angle. The coverage is increased to $98 \%$ of $4 \pi$ by $\mathrm{NaI}$ ( $\mathrm{Tl}$ ) endcaps. The direction of charged particles is measured in 3 double layers of proportional tube chambers. The Crystal Ball in its configuration at SPEAR has been described in detail elsewhere ${ }^{10)}$; at DORIS the main detector is unchanged. For the detection of hadronic events it is used as a calorimeter. The thickness of the NaI (TI) shell corresponds to 16 radiation lengths and to one interaction length for high energy pions. Therefore the distribution of energy deposited by charged hadrons peaks at around $200 \mathrm{MeV}$ due to non-interacting minimumionizing charged particles and has a long tail caused by nuclear interactions. Neutral, electromagnetically decaying hadrons deposit all their energy in the detector. 
The selection of hadronic events and the determination of the visible cross section was done with three different methods which gave compatible results. Two of the methods yielded results so rapidly that they were used to optimize the scan strategy during the run. In the following, one particular method is presented.

The events used in this analysis triggered the apparatus by passing a totalenergy threshold at about $1800 \mathrm{MeV}$. The pattern of energy deposition was required to be symmetric with respect to the interaction point in order to remove cosmic ray and beam-gas events. A minimum of 5 detected particles (charged or neutral) was required. The latter cut also removed events due to the QED processes $e^{+} e^{-} \rightarrow e^{+} e^{-}$and $e^{+} e^{-} \rightarrow \gamma \gamma$.

The contamination of the hadron sample due to cosmic ray and beam-gas events was measured with separated-beam runs which were distributed over the period of data taking and was found to be less than $2 \%$.

The luminosity was measured in two ways. Firstly, a small angle luminosity monitor is used, which detects Bhabha scattering events in the range of $6^{\circ}$ - $10^{\circ}$ of the scattering angle. Secondly, the main detector is used to identify Bhabha and $\mathrm{e}^{+} \mathrm{e}^{-} \rightarrow \gamma \gamma$ events in the angular range given by $|\cos \Theta|<0.85$. The two luminosities agreed very well and their ratio was constant within errors over the run period.

The resulting visible cross section is shown in Fig. 5. Runs without depolarization energy measurement were not used. The data points were fitted with the same function as in Fig. 4 (solid line). The resulting mass value is $(10022.8 \pm 0.5) \mathrm{MeV}$, t. $\mathrm{shere}$ the error is statistical only. The Gaussian width owing to the beam energy spread was found to be $8.1 \pm 0.5 \mathrm{MeV}$. This analysis is preliminary.

The two beam width results agree with the expected DORIS resolution ${ }^{4)}$. The two mass results are averaged to give $m^{\prime}\left(T^{\prime}\right)=(10023.1 \pm 0.4) \mathrm{MeV}$. In combining the errors of the two experiments, it was taken into account that they are partly correlated owing to the common errors on the beam energy. The difference between the average beam energy determined by depolarization and the actual mean value in each of the two interaction areas is estimated to be small. A more detailed calculation of this effect is under way, an upper limit at present is $\pm 0.25 \mathrm{MeV} /$ beam, resulting in 


$$
m\left(T^{\prime}\right)=(10023.1 \pm 0.4 \pm 0.5) \mathrm{MeV} .
$$

Using the Novosibirsk value ${ }^{3)}$ for $\mathrm{m}(\mathrm{T}),(9459.7 \pm 0.6) \mathrm{MeV}$, this result fixes the T'-T mass difference to

$$
\Delta m=(563.4 \pm 0.9) \mathrm{MeV} .
$$

This result is compared to previous ones in Table $l$.

TABLE 1

The mass difference $m\left(T^{\prime}\right)-m(T)$ as determined by different

experiments (in MeV).

\begin{tabular}{|l|l|}
\hline DASP 2 11) & $556 \pm 10$ \\
LENA 12) & $560 \pm 10$ \\
CLEO 13) & $560.7 \pm 0.8 \pm 3.0$ \\
CUSB 14) & $559 \pm 1 \pm 3$ \\
ARGUS 1) & $561 \pm 3$ \\
this experiment & $563.4 \pm 0.9$ \\
\hline
\end{tabular}

\section{FIGURE CAPTIONS}

Fig. 1 Block diagram of the DORIS depolarizer.

Fig. 2 Schematic view of the DORIS polarimeter.

Fig. 3 Example of a depolarization curve. Horizontal error bars indicate the sweep range of the frequency generator.

Fig. 4 The visible hadronic cross section over the $T$ ' resonance as measured in ARGUS.

Fig. 5 The visible hadronic cross section over the T' resonance as measured in the Crystal Ball detector. 


\section{REFERENCES}

l) H. Albrecht, G. Drews, H. Hasemann, A. Philipp, $\cdot$. Schmidt-Parzefall, H. Schröder, H.D. Schulz, F. Selonke, R. Wurth (DESY, Hamburg, Germany);

A. Drescher, B. Gräıe, H. Hofmann, A. Markees, U. Matthiesen, J. Spengler,

D. Hegener (Institut für Physik, Universität Dortmund, Germany);

R. Heller, K.R. Schubert, J. Stiewe, R. Waldi, S. Weseler (Institut für Hochenergiephysik, Universität Heidelberg, Germany);

K. Edwards, H.R. Frisken, Ch. Fukunaga, M. Goddard, P. Kim, R.S. Orr, P.M. Patel, J.D. Prentice, H. Seywerd, T.-S. Yoon (Institute of Particle Physics, Canada); R. Ammar, D. Coppage, R. Davis, N. Ktsak (University of Kansas, Lawrence, USA); P. Boeckmann, L. Jönsson, Y. Oku (Institute of Physics, University of Lund, Sweden); A. Arefiev, M. Danilov, V. Lubimov, V. Matveev, V. Nagovitsin, Yu. Semenov, V. Shevchenco, V. Soloshenko, V. Sopov, V. Tchernyshew, I. Tichomirov, V. Tchistilin, Yu. Zaitsev (Institute of Theoretical and Experimental Physics, Moscou, USSR); R. Childers, C.I. Darden and H. Gennow (University of South Carolina, USA), ARGUS 83-01.

2) C. Edwards, C. Peck, F. Porter, P. Ratoff (California Institute of Technology, Pasadena, USA); I. Brock, A. Engler, B. Kraemer, D. Marlow, F. Messing, D. Prindle, B. Renger, C. Rippich (Carnegie-Mellon University, Pittsburgh, USA); Z. Jakubowski, B. Niczyporuk, G. Nowak, T. Skwarnicki (Cracow Institute of Nuclear Physics, Cracow, Poland); J.K. Bienlein, S. Cooper, B. Gomez, T. Kloiber,. . Koch, M. Schmitz, H.-J. Trost, P. Zschorsch (Deutsches Elektronen-Synchrotron DESY, Hamburg, Germany); D. Antreasyan, J. Irion, K. Strauch, D. Hilliams (Harvard University, Cambridge, USA); D. Besset, R. Cabenda, M. Cavalli-Sforza, R. Cowan, D. Coyne, C. Netuman- Holmes (Princeton University, Princeton, USA); E. Bloom, R. Chestnut, R. Clare, J. Gaiser, G. Godfrey, S. Leffler, H. Lockman, S. Lotse, K. Hacker (Stanford Linear Accelerator Center, Stanford, USA); D. Gelphman, R. Hofstadter, I. Kirkbride, R. Lee, A. Litke, T. Matsui, B. Pollock, J. Tompkins (Stanford University, Department of Physics and HEPL); G. Folger, B. Lurz, U. Volland, H. Wegener (Universität Erlangen-Nürnberg, Erlangen, Germany); A. Cartacci, G. Conforto, D. de Judicibus, B. Monteleoni, P.G.Pelfer (INFN and University of Firenze, Italy); A. Fridman, F. Heimlich, R. Lekebusch, P. Lezoch, H. Maschmann, R. Nernst, A. Schwarz, D. Sievers, U. Strohbusch (Universität Hamburg, I. Institut für Experimentalphysik, Hamburg, Germany); A. König, J. Schotanus, R.T. Van de Halle, H. Halk (University of Nijmegen, The Netherlands); S. Keh, H. Kilian, K.Königsmann, M. Scheer, P. Schmitt (Universität lJürzburg, Germany); D. Aschman (University of Cape Tot.sn, South Africa).

3) A.S. Artamonov et al., Phys.Lett. 118B (1982) 225

4) K. Hille, DESY 81-047 (1981)

5) A.A. Sokolov and I.M. Ternov, Sov.Phys. Dok1. 8(1964) 1203 
6) S.I. Serednyakov et al., JETP 44 (1976) 1063

7) R. Neumann and R. Rossmanith, Nucl. Instr. Meth. 204 (1982) 29

8) D.P. Barber et al., DESY M-83-15 (1983)

9) J.D. Jackson and D.L. Scharre, Nucl. Instr. Meth. 128 (1975) 13

10) M. Oreglia, Ph.d. thesis, Stanford University, SLAC-236 (1980)

M. Oreglia et al., Phys.Rev. D25 (1982) 2259

J. Gaiser, Ph.D. thesis, Stanford University, SLAC-255 (1982)

11) C.H.Darden et al. (DASP2), Phys.Lett. 78B (1978) 364 and

H. Albrecht et al., (DASP2), Phys.Lett. 116B (1982) 283

12) J.K. Bienlein et al. (LENA), Phys.Lett.78B (1978) 360

13) D. Andrews et al. (CLEO), Phys. Rev. Lett. 44 (1980) 1108

14) T. Boehringer et al. (CUSB), Phys.Rev.Lett. 44 (1980) 1111. 


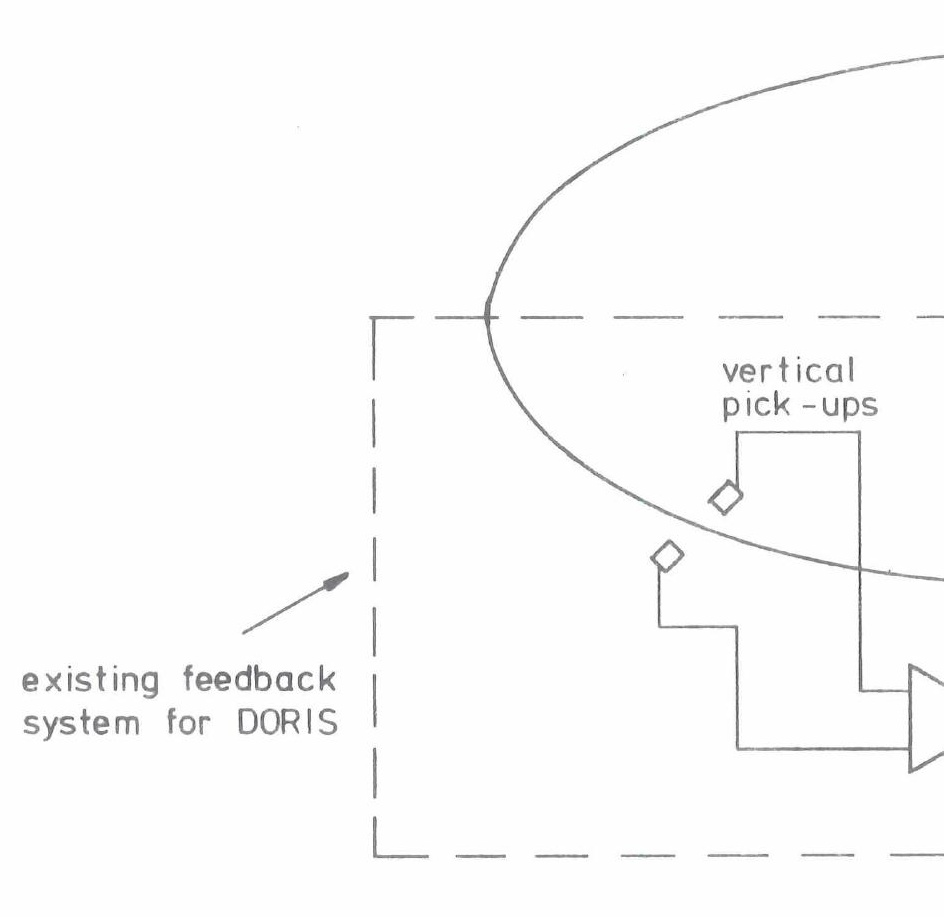




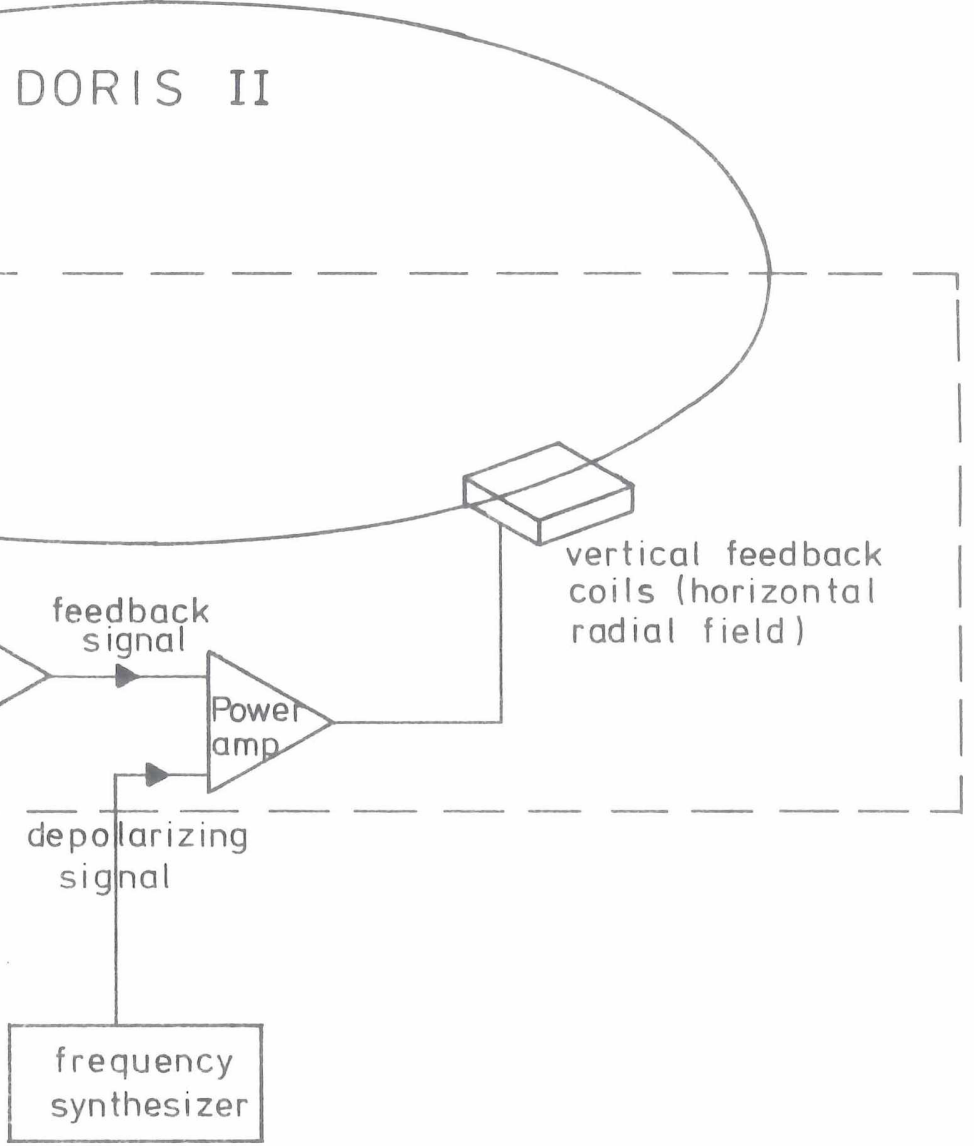

Fig. 1 


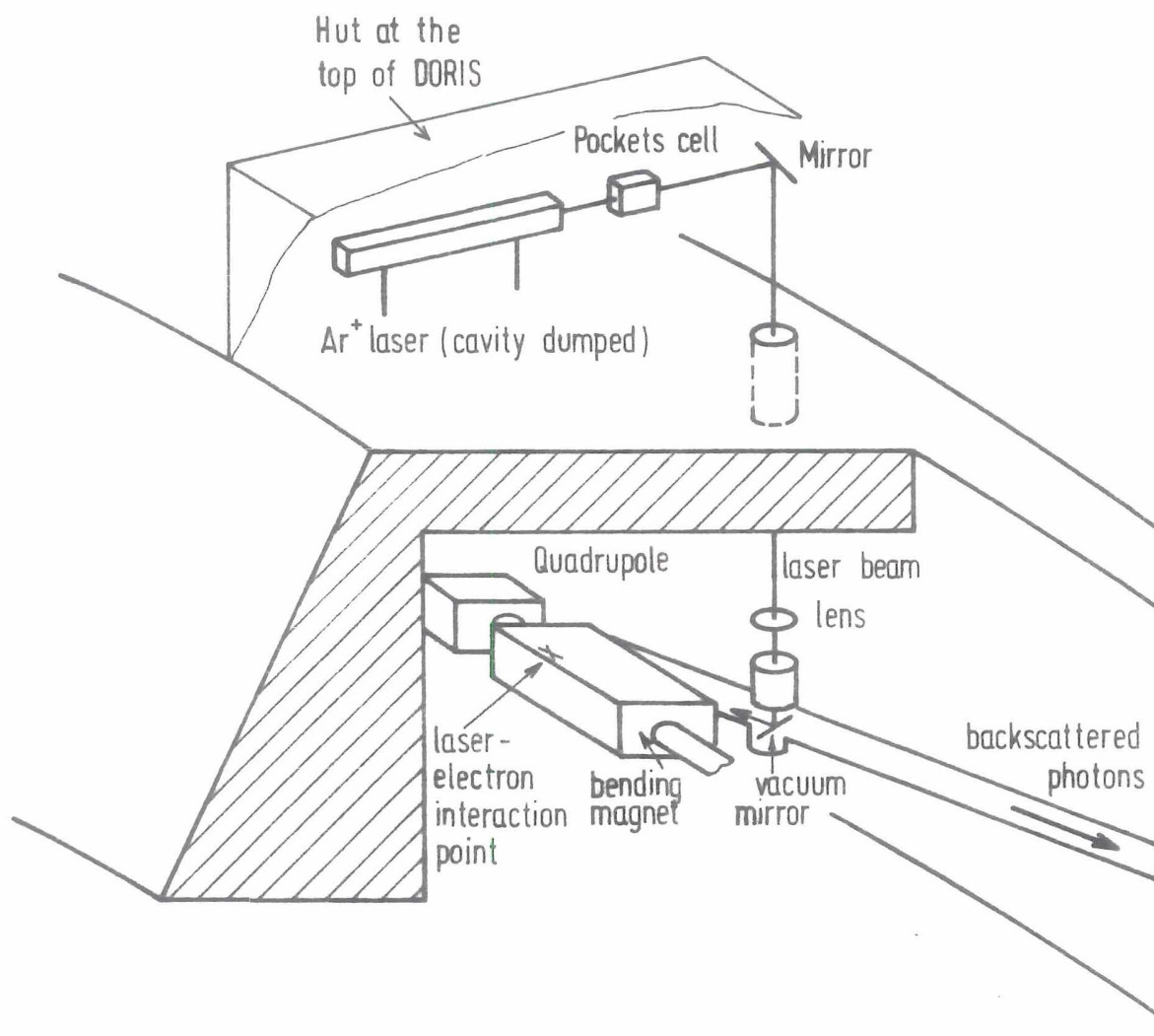

Fig. 2 


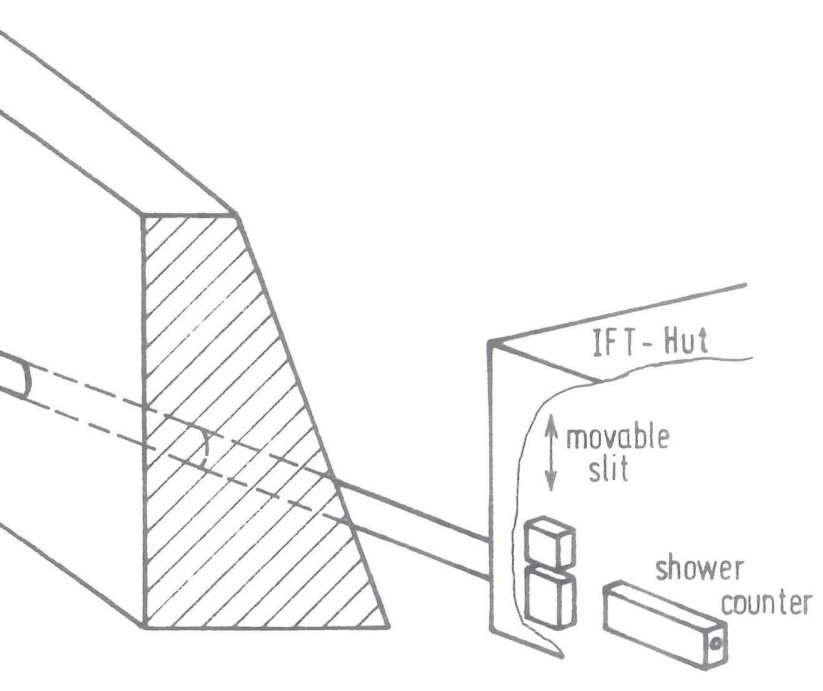




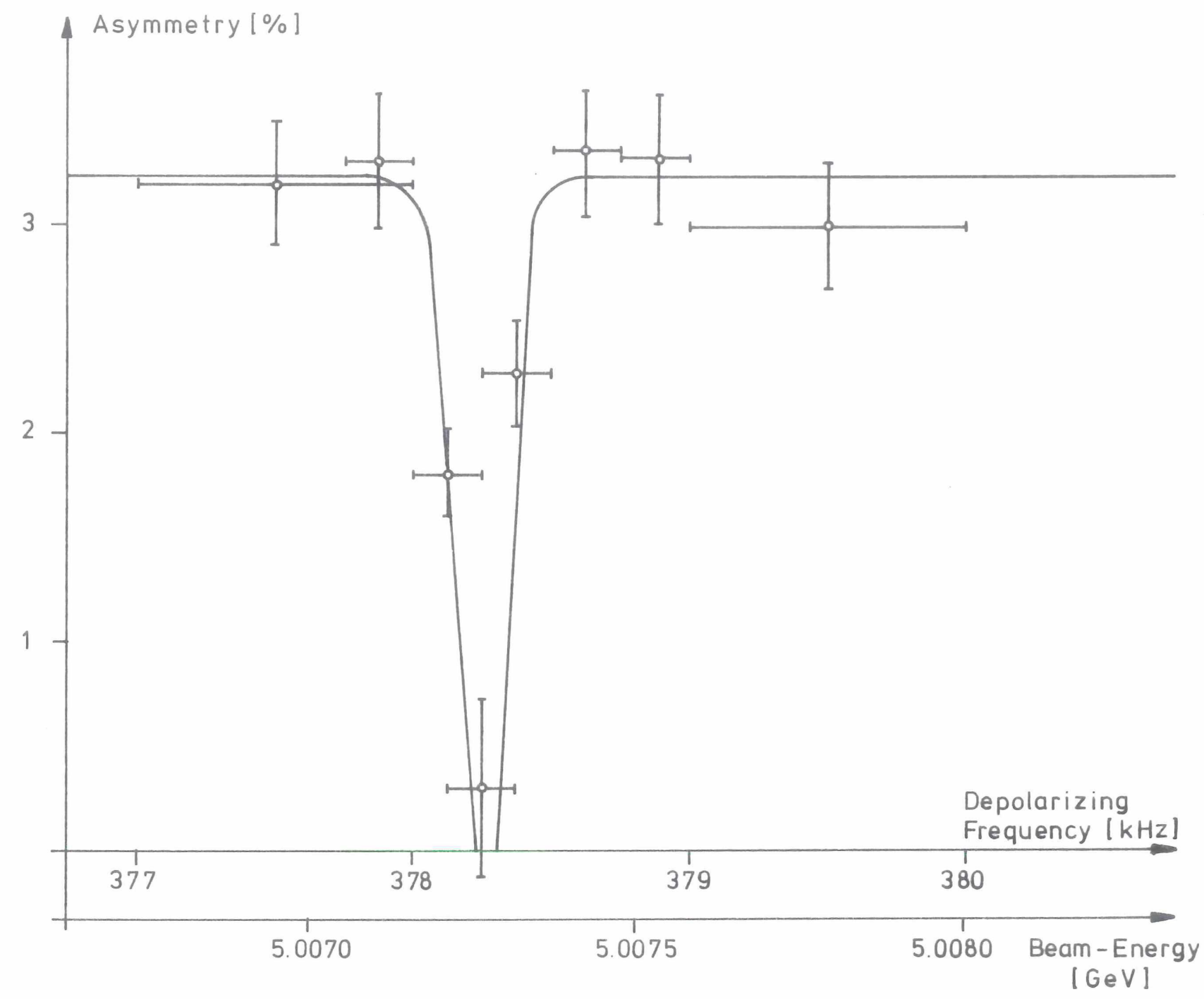

Fig. 3 


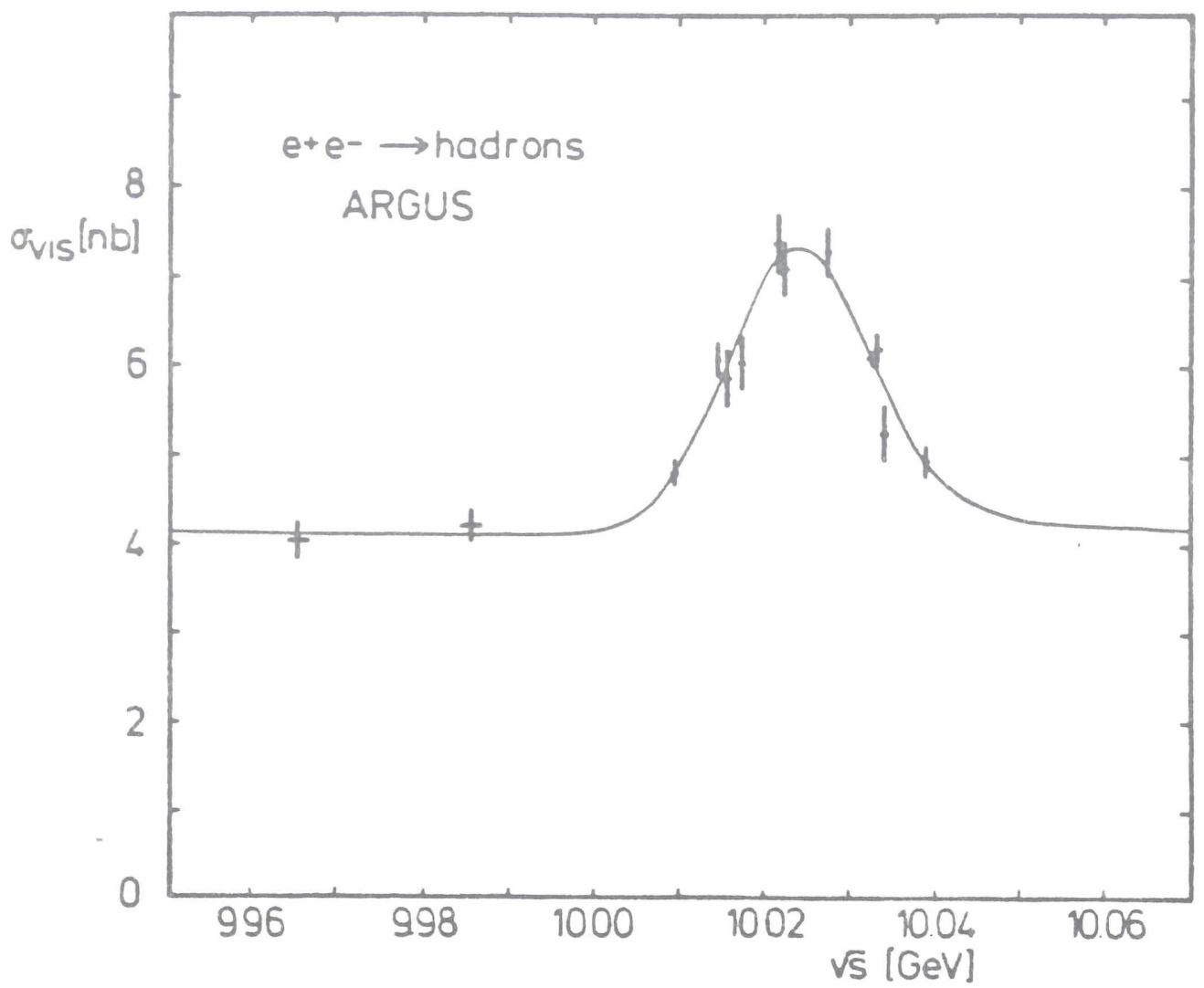

Fig. 4

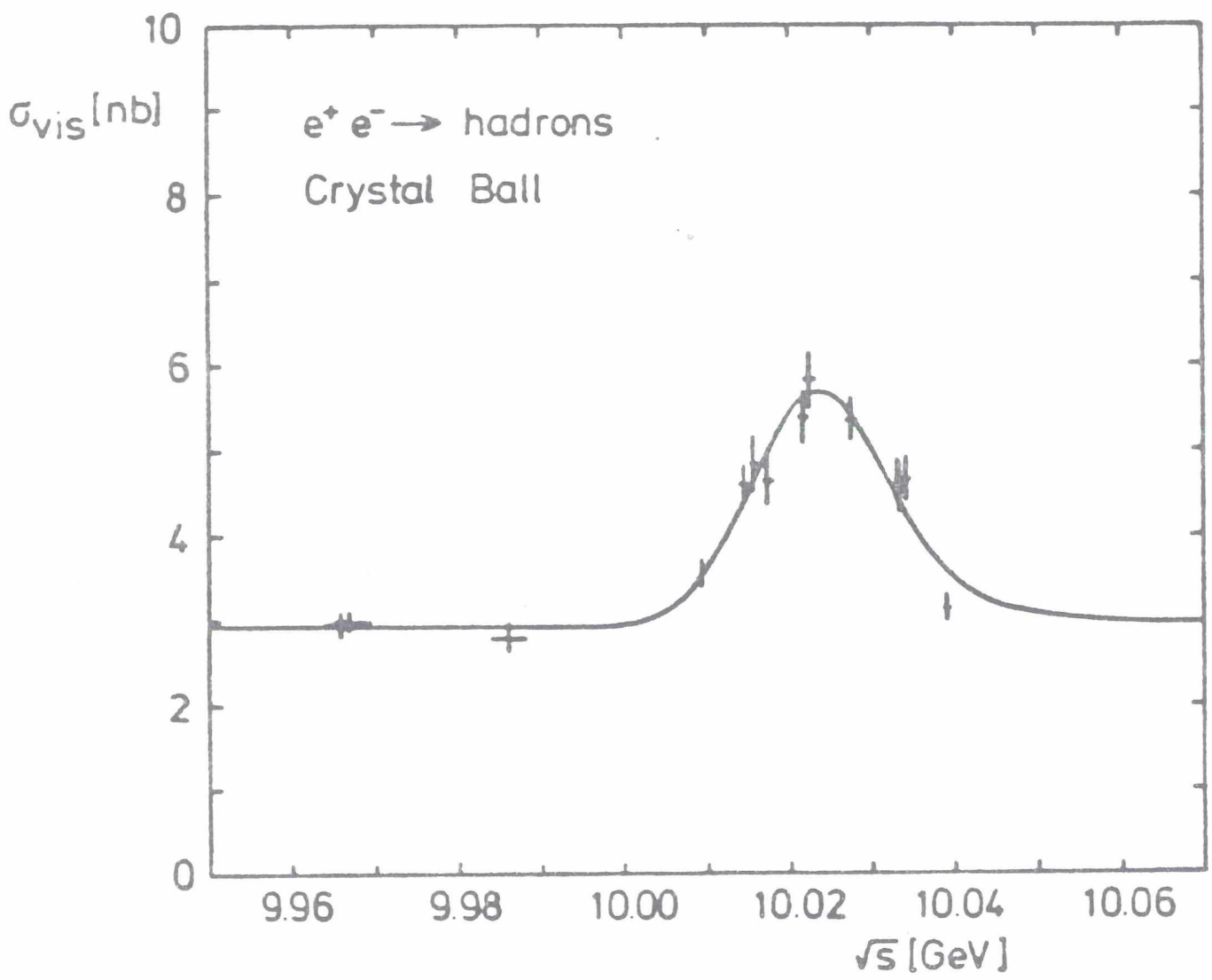

Fig. 5 